\title{
Über \\ das Verhalten der durchsichtigen Augenmedien gegen ultraviolette Strahlen.
}

Von

Dr. T. Takamine und Dr. S. Takei.

(Hierzu Tafel V und VI.)

\section{Einleitung.}

Die Frage, ob die ultravioletten Strahlen überhaupt zur Netzhaut gelangen, und wenn es geschieht, warum sie uns unsichtbar bleiben, wurde schon von vielen Seiten auf experimentellen Wege zu beantworten versucht. Merkwürdigerweise sind die diesbezüglichen Angaben selbst in den neuesten Lebr- und Handbüchern der Physiologie sehr mangelhaft. Die Frage hat offenbar Augenärzte mehr interessiert als Physiologen. Die Sache ist jedoch von solcher Wichtigkeit, dass sie in jedem physiologischen Lehrbuch erwähnt werden sollte. Bezüglich der genaueren Literaturangaben auf die Arbeit von Birch-Hirschfeld ${ }^{1}$ ), die Wirkung der ultravioletten Strahlen auf das Auge" verweisend, wollen wir uns hier auf die Erwähnung der seither erschienenen Arbeiten beschränken.

Man hatte die Frage überhaupt von zwei Seiten angegriffen: einerseits durch das Studium der Veränderungen in der Netzhaut, hervorgerufen durch die ultravioletten Strahlen, anderseits durch die Untersuchung, ob diese Strahlen dureh die Augenmedien absorbiert werden oder nicht, bevor sie die Netzhaut erreichen. Die Resultate, zu denen man hierdurch gelangte, waren ebenso verschieden wie die angewandten Methoden selbst, die je nach dem derzeitigen Stande der Wissenschaft verschieden gewählt wurden.

1) Birch-Hirschfeld, Die Wirkung der vitravioletten Strahlen auf das Auge. Arch. f. Ophthalm. Bd. 58 S. 469. 1904. 
Seitdem Schulek ${ }^{1}$ ) die praktische Seite der Frage berührte und behauptete, dass die ultravioletten Strahlen eine wesentliche Rolle bei der Entstehung des menschlichen Kataraktes spielen, folgten zahlreiche Untersuchungen von seiten der Augenärzte. Besonders sind die Arbeiten von Birch-Hirschfeld ${ }^{2}$ ) sowie von Schanz und Stockhausen ${ }^{3}$ ) zu nennen.

Die letzteren Autoren haben die Lichtstrablen je nach ihrer Wirkung auf das Auge und je nach ihren Wellenlängen in vier Gruppen eingeteilt, von denen nur die erste Gruppe uns sichtbar ist. Diese Gruppe umfasst die Strahlen von der Wellenlänge 760-400 $\mu \mu$ (rot bis violett), die ohne Transformation zur Netzhaut gelangen und von uns wahrgenommen werden. Zur zweiten Gruppe gehören solche von $400-350 \mu \mu$, die die Linse lavendelgrau erscheinen lassen, wenn sie sie treffen. Sie gelangen nicht direkt zur Netzhaut, werden erst sichtbar entweder, wenn die Linse entfernt wird, oder wenn die Strahlen des sichtbaren Spektrums stark abgeschwächt werden. Die dritte umfasst Strablen von $350-300 \mu \mu$. Diese dringen in das Auge ein, erreichen aber nicht die Netzhaut, sondern werden von der Linse absorbiert. Die zur vierten Gruppe gehörenden Strahlen haben die Wellenlängen von unterhalb $300 \mu \mu$. Sie passieren nicht mehr die Hornhaut, verursachen vielmehr Entzündung des äusseren Auges.

Die Untersuchungsmethode der genannten Autoren war die, dass Hornhaut, Linse und Glaskörper abwechselnd vor die Spalte des Spektroskops gebracht und 2-120 Sekunden lang den zu untersuchenden Strahlen ausgesetzt und photographiert wurden. Am Ende des Versuches wurde dann die Absorption des ganzen kombinierten Systems des Auges untersucht. Mit den auf diese Weise gewonnenen Ergebnissen stimmen die Resultate von Birch-Hirschfeld im wesentlichen überein, die in noch exakterer Weise gewonnen wurden.

Hallauer ${ }^{4}$ fand bei einer grösseren Versuchsreihe die Absorption kurzwelliger Strahlen durch die menschliche Linse im all-

1) Schulek, Ungar. Beitr. zur Augenheilk. Bd. 2 S. 467.

2) Birch-Hirschfeld, Zur Beurteilung der Schädigung des Auges durch kurzwelliges Licht. Zeitsclir. f. Augenheilk. Bd.21 S. 385. 1909.

3) Schanz und Stockhausen, Über die Wirkung der ultravioletten Strahlen auf das Auge. Arch. f. Ophthalm. Bd. 69 S. 452.1908.

4) Halla u er, Über die Absorption von. kurzweligem Licht durch die menschliche Linse. Klin. Monatsbl. f. Augenheilk. Jahrg. 47 S. 721.1909. 
gemeinen abhängig vom Lebensalter, von der Konstitution des Körpers und von individuellen Verschiedenheiten der Linse (Dicke, Färbung, Konsistenz). Die Linse des Kindes und des jugendlichen Menschen absorbierte Strahlen bis zu $400 \mu \mu$. Nach dem zwanzigsten Lebensjahre trat eine Verschiebung des Absorptionsvermögens bis auf etwa $377 \mu \mu$ ein, welches dann bei zunehmendem Alter auf $400-420 \mu \mu$ anstieg. Zehrende Krankheiten wie Tuberkulose und Karzinom setzen den Wert bis auf $375 \mu \mu$ herab.

Ganz neuerdings hat E. K. Martin ${ }^{1}$ ) die Wirkung der ultravioletten Strahlen auf das Auge des Kaninchens studiert. Als Lichtquelle diente eine Eisenbogenlampe, deren Strahlen mittels eines Quarzspektrographs aufgenommen wurden. Die Hornhaut liess alle Strablen nicht durch, die kürzere Wellenlängen besassen als $295 \mu \mu$. Bei der Linse begann die Absorption bei $400 \mu \mu$ und wurde jenseits von $350 \mu \mu$ vollständig. Der Glaskörper zeigte ein breites Absorptionsband mit schlecht begrenzten Rändern, das sich zwischen 280-250 $\mu \mu$ erstreckte. Also waren alle Medien des Auges nur für die Strahlen mit der Wellenlänge von $660-400 \mu \mu$ permeabel.

Die Einwirkung der kurzwelligen Strahlen auf die Netzhaut bat man einerseits nach ihrer Sichtbarkeit, anderseits nach der histologischen Veränderung der Retina beurteilt, die sie auf derselben hinterlassen. Als Birch-Hirschfeld die Sichtbarkeit der ultra* violetten Strahlen an dem Auge mit und ohne Linse verglich, fand er, dass die Fähigkeit, die Existenz ultravioletter Strahlen zu erkennen, am linsenlosen Auge beträchtlich überwiegt. Auch konstatierte er, dass das linsenhaltige Kaninchenauge nach $1 / 2-1$ Stunde langer Bestrahlung mit dem ultravioletten Spektrallicht einer Bogenlampe normale Netzhautstruktur zeigte. War das Auge dagegen linsenlos, so fand er nach gleicher Prozedur Veränderungen in den Ganglienzellen, in den Körnern der inneren und äusseren Körnerschicht, die in Chromatinverlust, Vakuolenbildung und Formveränderung der Zellen bestanden.

Neuerdings hat er mit N. Inou e ${ }^{2}$ ) die Versuche wiederholt, indem er das linsenlose Taubenauge intensiven ultravioletten Strahlen

1) E. K. Martin, The effects of ultra-violet rays on the eye. Nature Nr. 2212 vol. 89.1912.

2) Birch-Hirschfeld und In oue, Weitere Versuche über die Wirkung des ultravioletten Lichtes auf die Netzhaut. PfIüger's Arch. Bd. 136 S. 595. 1910. 
aussetzte. Sie fanden dabei die vorwiegende Beteiligung der inneren Netzhautschichten, deutlicher als bei den früheren Versuchen. Als Lichtquelle benutzten sie diesmal die Schott'sche Uviollampe, eine Quecksilberdampflampe, deren Spektrum bis auf etwa $253 \mu \mu$ Wellenlänge reicht.

\section{Eigene Untersuchung.}

Unsere Methode des Experimentes war ganz ähnlich der von Birch-Hirschfeld. Als Lichtquelle haben wir eine Quarzquecksilberdampflampe von $\mathrm{Heräus}$ angewendet, die durch Akkumulatoren von 100 Volt in Funktion gesetzt wurde. Der elektrische Strom von 2,5 Ampère wurde mit dem Rheostat und Amperemeter von Siemens kontrolliert. Die Lampe wurde in einen grossen, auf der einen Seite mit einem ca. 8 qcm grossen Fenster versehenen Holzkasten gesetzt. Ein Fu ess'scher Quarzspektrograph mit einem Cornus'schen Prisma wurde so vor die Lampe gestellt, dass die Spalte desselben gerade gegen das Fenster des Holzkastens gerichtet war. Die Einrichtung des Apparates erlaubte es, durch revolverartige Drehung des Plattenhalters um die vertikale Achse einen beliebigen Teil oder auch die ganze Länge des Spektrums scharf einzustellen. Ebenso konnte man auch die Platte durch Schraubenvorrichtung auf- und abbewegen und nach beliebiger Expositionsdauer wieder neue Stellen einschalten.

Die Experimente wurden in folgender Weise angestellt: Nach Anzündung der Lampe wurde eine Zeitlang gewartet, bis das Licht und die Stromstärke konstant wurden. Nun brachten wir das zu untersuchende Material direkt vor die Spalte des Apparates und öffneten dieselbe durch Drehen der vor der Spalte gelegenen Drehscheibe. Die Exponierung dauerte in der Regel 5 Minuten, und nach Verschluss der Spalte wurde die photographische Platte vertikal verschoben, um neue lichtempfindliche Stellen zu erhalten. Dann wechselten wir das Material, um den gleichen Prozess zu wiederholen. Die Breite der Spalte blieb während des ganzen Experimentes hindurch $0,057 \mathrm{~mm}$ weit. Zur photometrischen Kontrolle des Experimentes wurden nach einer Reihe von Versuchen Photogramme aufgenommen, ohne Einschaltung des Materials, oder indem man nur die Quarzplatteu vor die Spalte legte.

Die brechenden Medien des Auges wurden in folgender Weise vorbereitet. 
1. Die Hornhaut wurde über den Querschnitt eines kurzen Glasröhrchens gespannt und dicht vor die Spalte des Spektrographs gelegt. Zuweilen kamen trotz Vorsicht einige unvermeidliche Febler zum Vorschein, dass z. B. durch kleine Risse oder Faltenbildung die Linien des Spektrums gebrochen erschienen.

2. Der Glaskörper wurde in ein kleines Gefäss mit zwei parallelen Wänden aus Quarzplatten gelegt. Die Dicke der Wand betrug $1 \mathrm{~mm}$ und der Abstand der beiden Wände $8 \mathrm{~mm}$.

3. Linse. Bei der Linse werden die durchgehenden Strahlen, wie Birch-Hirschfeld schon bemerkte, nicht nur durch Absorption, sondern durch Refraktion modifiziert, wenn man die Linse in ihrer natürlichen Form vor die Spalte setzen wollte. Daher legten wir sie zwischen zwei parallele Quarzplatten von $1 \mathrm{~mm}$ Dicke und suchten durch leichtes Drücken die Krümmung ihrer Oberfläche zu korrigieren.

Zur Untersuchung kamen:

1. Augen einer 37jäbrigen Frau (aus frischem Leichenmaterial),

2. " vom Ochsen,

3. " "Kaninchen,

4. " der Katze,

5. " vom Hund,

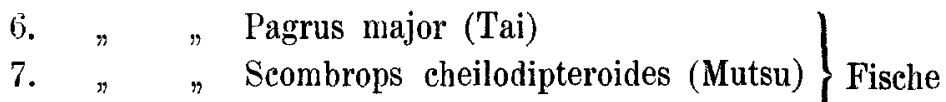

8. " " Sebastodes matsubarae (Ako)

9. " " Syrnium spec. (Eule)

10. " " Bubo maximus (Mimizuku) $\}$ Vögel

Zur photographisehen Aufnahme der Spektrallinien kamen folgende Platten zur Anwendung. Für Fig. 1, 3, 4, 5, 6 und 8 „Lion: by the Imperial Dry Plate Co., London with speed gradation of 200 in Hurter \& Driffield unit"; für Fig. 2, 7 und 9 "Lion orthochromatic plate".

Zur Entwicklung der Platten haben wir folgende Lösungen während der ganzen Untersuchung angewandt:

a) $20 \%$ ige Lösung von Natrium sulfurosum,

b) $20 \%$ ige Lösung von Natrium carbonicum,

c) ein Gemisch von a und bāa $100 \mathrm{ccm}$ mit $1 \mathrm{~g}$ Kal. bromat.,

d) $30 \%$ ige Lösung von Acid. pyrogallicum.

Beim Gebrauch wurden die Lösungen $c$ und $d$ im Verhältnisse von 10:1 genommen und mit vierfachem Wasser verdunnt. 
Zur Bestimmung der Lage der Spektrallinien des Quecksilbers wurde die Photographie von $K \ddot{u} c h$ und Retschinsky zu Rate gezogen. (Ann. d. Physik, 22 Taf. 1. 1907.)

\section{Schluss.}

Von den drei durchsichtigen Medien des Auges, nämlich der Hornhaut, dem Glaskörper und der Linse, hat die Linse am meisten die Fähigkeit, kurzwellige Strahlen zu absorbieren. Bevor wir nun in die Einzelheiten näher eingehen, sei es gleich erwähnt, dass es individuelle Sch wankungen des Absorptionsorades gibt, die sich zwischen $20-30 \mu \mu$ bewegen, so dass es nicht wichtig zu sein scheint, die Wellenlänge des Spektrums an jeder Stelle mit einer Genauigkeit von wenigen Ångström - Einheiten zu berücksichtigen. Die kurzwelligen Spektrallinien des Quecksilbers waren in ditsem Falle genügend vorhanden. Die Frage, welche Differenz zwischen dem lebenden und toten Auge in bezug auf die Absorption des Lichtes vorhanden ist, ist schwer zu beantworten. Einige Autoren haben geglaubt, aus der Fluoreszenz der Linse im Auge lebender Tiere (Taube, Kanincben usw.) durch ultraviolette Strahlen einen Schluss auf die vorhandene Lichtabsorption ziehen zu können, doch scheint es uns gewagt, aus diesem Phänomen allein auf die Absorption zu schliessen.

Was dje Absorptionsfähigkeit einzelner Augenmedien betrifft, so können wir aus unseren Experimenten folgendes schliesen:

a) Hornhaut. Die Absorption der ultravioletten Strablen durch die Hornhant war bei allen Tierklassen fast immer gleich stark. Die minimale Wellenlänge der durchgelassenen Lichtstrahlen schwankte zwischen 297 und $280 \mu \mu$. Dieser Wert weicht von dem von Birch-Hirschfeld am Kalbs- und Schafsauge enthaltenen nicht wesentlich $\mathrm{ab}$, da dieser $306 \mu \mu$ betrug, in Übereinstimmung mit der früheren Beobachtung von Schanz und Stockhausen.

b) Glaskörper. Dieser verhielt sich in den meisten Fällen auch fast gleich wie die Hornhaut und absorbierte die Strahlen fast. in gleichem Grade. Die kleinste Wellenlänge lag zwischen $297 \mu \mu$ und $280 \mu \mu$. Merkwürdigerweise zeigte der Glaskörper des Hundes und der Katze eine Abweichung hiervon, indem derselbe die kurzwelligen Strahlen bis $265 \mu \mu$ durchliess. Die gleiche Eigentümlichkeit von schwächerer Absorption kam beim Versuch mit der Linse dieser zwei Tierspezies zum Vorschein. Birch-Hirschfeld gibt den 
Wert von $300 \mu \mu$ für den Glaskörper des Schweines und des Schafes an.

c) Linse. Das zu unseren Versuchen benutzte Material können wir je nach dem Grade der Absorption in drei Klassen einteilen:

1. Die schwächste Absorption zeigten die Linsen von Hund und Katze : $313 \mu \mu$;

2. schwache Absorption zeigten die Linsen vom Ochsen, Kaninchen, Eule, Scombrops cheilodipteroides (Mutsu) und Sebastodes matsubarae (Ako): $363 \mu \mu$;

3. starke Absorption zeigten die Linsen von Mensch und Pagrus major (Tai): $405 \mu \mu$.

Man sieht hieraus, dass die Linsen der Katze und Hunde, die nachts ihre Dienste leisten, am meisten für kurzwellige Strahlen durchlässig sind.

Die Linsen der Tiefseefische wie die der Scombrops und der Sebastodes besitzen eine ähnliche Durchlässigkeit wie die der Eule. Die ersteren leben in der Tiefe des Meeres, wo die Lichtstrahlen nur schwer durchdringen, letztere geht nachts auf Raub aus. Ochsen und Kaninchen können auch wahrseheinlich bei schwacher Beleuchtung sehen.

Ausser dieser physikalischen Eigensehaft der Augenmedien wird allerdings die Feinheit des Tast-, Gehör- und Geruchssinnes ebenfalls dazu beitragen, dass die Tiere im Dunkeln sich zurecht finden.

Interessant ist auch, dass die Linse des Pagrus major, der sich in relativ seichter Stelle des Meeres aufhält, eine fast gleiche Durchlässigkeit besitzt wie die des Menschen.

Zum Vergleich seien hier einige von anderen Autoren erhaltene Werte für die Linse zitiert:

$\left.\begin{array}{c|c|c|c}\hline \begin{array}{c}\text { Name } \\ \text { der Autoren }\end{array} & \begin{array}{c}\text { Linse } \\ \text { vom }\end{array} & \text { Minimum } \lambda & \text { Lichtquelle } \\ \hline \text { Brücke }{ }^{\mathbf{1}} \ldots . . & \text { Ochsen } & 370 & \text { Sonnenlicht } \\ \text { de Chardonnet }{ }^{2} \text { ) } & \text { Menseh }\left\{\begin{array}{c}\text { Absorption beginnt bei } \\ 397 \text { und wird voll- } \\ \text { ständig bei 381-372 }\end{array}\right.\end{array}\right\} \begin{gathered}\text { Elektrisches Bogen- } \\ \text { licht }\end{gathered}$

1) Brücke, Über das Verhalten der optischen Medien des Auges gegen Licht- und Wärmestrahlen.' Arch. f. Anat. u. Physiol. 1845 S. 262.

2) de Chardonnet, Pénétration des radiations actiniques dans l'œil de I'homme et des animaux vértebrés. Compt. rend. l'acad. des sciences t. 96 p. 441 . 1896 . 


\begin{tabular}{|c|c|c|c|}
\hline $\begin{array}{c}\text { Name } \\
\text { der Autoren }\end{array}$ & $\begin{array}{l}\text { Linse } \\
\text { vom }\end{array}$ & Minimum $\lambda$ & Lichtquelle \\
\hline Schulek. . . & Mensch & $\begin{array}{l}\text { Absorption beginnt bei } \\
397 \text { und wird voll- } \\
\text { ständig bei } 373\end{array}$ & Sonnenlicht \\
\hline $\begin{array}{c}\text { Schanz u. Stock- } \\
\text { hausen... }\end{array}$ & Mensch & 350 & Bogenlampe \\
\hline $\begin{array}{l}\text { Birch-Hirsch- } \\
\text { feld..... }\end{array}$ & $\begin{array}{l}\text { Schwein } \\
\text { Kalb } \\
\text { Rind } \\
\text { Kaninchen }\end{array}$ & $\begin{array}{l}330( \pm 15) \\
328( \pm 12) \\
370-400 \\
330-390\end{array}$ & $\begin{array}{c}\text { Elektrischer Funken } \\
\text { zwischen Zinkelek- } \\
\text { troden in Stickstoff- } \\
\text { atmosphäre }\end{array}$ \\
\hline Hallauer... & Mensch & $375-400$ & Eisenlicht \\
\hline E. K. Martin & Kaninchen & $\begin{array}{c}\text { Absorption beginnt bei } \\
400 \text { und wird komplett } \\
\text { bei } 350\end{array}$ & Eisenbogenlampe \\
\hline
\end{tabular}

\section{Der Brechungsindex der Augenmedien.}

Zur Bestimmung des Brechungsexponenten von den zur Untersuchung gekommenen Augenmedien haben wir den Refraktometer von $\mathrm{Abbe}$ benutzt. Die Versuche wurden bei einer Zimmertemperatur von $17^{\circ} \mathrm{C}$. bis $20^{\circ} \mathrm{C}$. angestellt. Um die experimentell erhaltenen Zahlen auf $18^{\circ} \mathrm{C}$. zu reduzieren, wurde nur die Änderung des Brechungsexponenten des Wassers berücksichtigt, welches den Hauptbestandteil der Augenmedien bildet. Aus der angeführten Tabelle 1 geht hervor, dass der Index für Glaskörper trotz der Versehiedenheit der Tierspezies auffallend gut übereinstimmt.

\section{Literatur.}

1) Birch-Hirschfeld, Die Wirkung der ultravioletten Strahlen auf das Auge. Arch, f. Ophthalm. Bd. 58 S. 469.1904.

(2) Schulek, Ungar. Beitr. zur Augenheilk. Bd. 2 S 467.

3) Schanz und Stockhausen, Über die Wirkung der ultravioletten Strablen auf das Auge. Arch. f. Ophthalm. Bd. 69 S. 452.1908.

4) Birch-Hirschfeld, Zur Beurteilung der Schädigung des Anges durch kurzwelliges Licht. Zeitschr. f. Augenheilk. Bd. 21 S. 385.1909.

5) Hallauer, Ü̈ber die Absorption von kurzwelligem Licht durch die menschliche Linse. Klin. Monatsbl. f. Augenheilk. Jahrg. 47 S. 721.1909. 
Über das Verhalten der durchsichtigen Augenmedien etc.

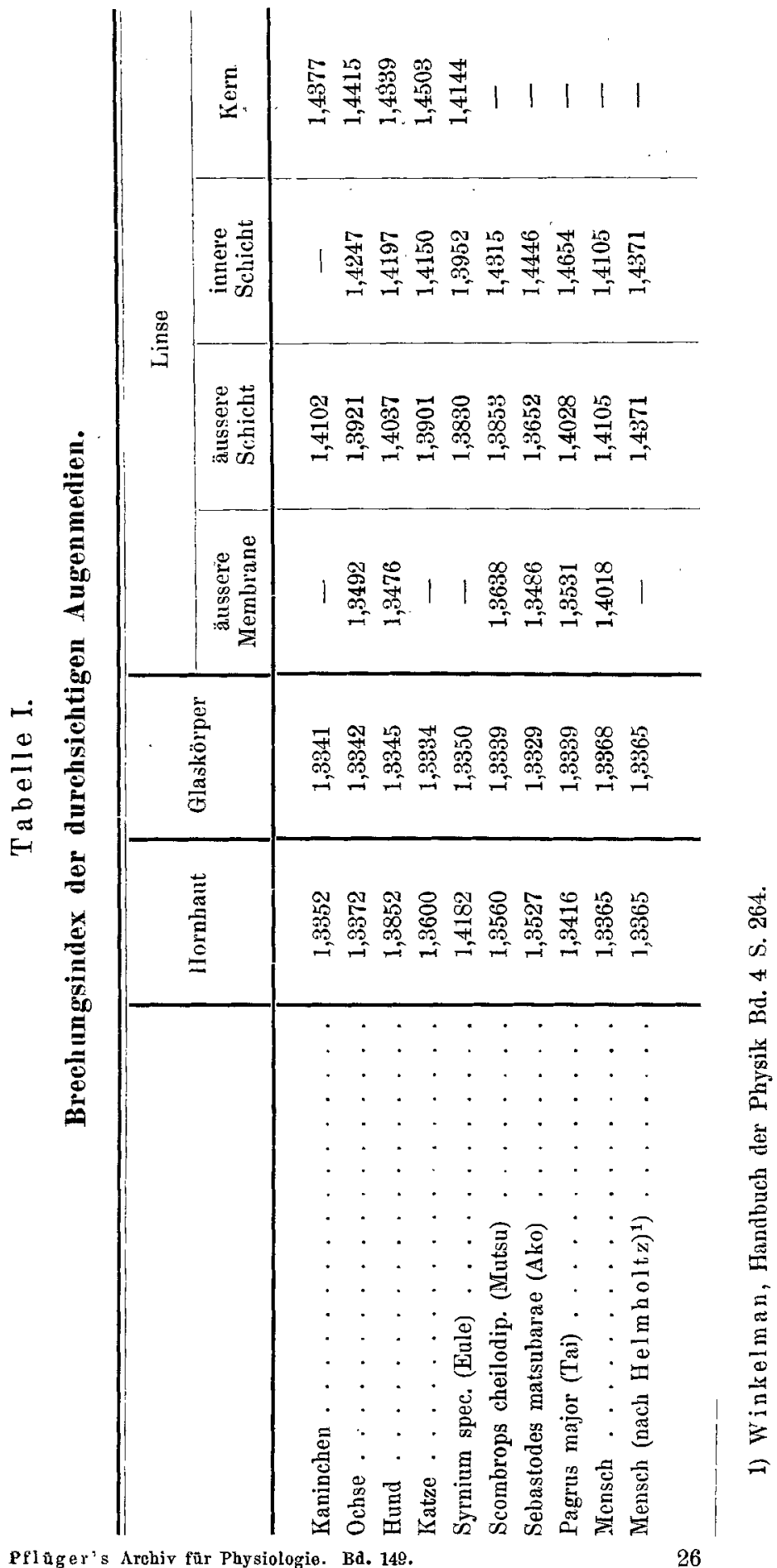


388 T. Takamine und S. Takei: Über das Verhalten ete.

6) E. K. Martin, The effects of ultra-violet rays on the eye. Nature No. 2212 vol. 89. 1912.

7) Birch-Hirschfeld und Inoue, Weitere Versuche über die Wirkung des ultravioletten Lichtes auf die Netzhaut. P flü ge r's Arch. Bd. 136 S. 595. 1910.

8) Brücke, Über das Verhalten der optischen Medien des Auges gegen Licht- und Wärmestrahlen. Arch. f. Anat. u. Physiol. 1845 S. 262.

9) de Chardonnet, Pénétration des radiations actiniques dans l'œil de l'homme et des animaux vértébrés. Compt. rend. de l'acad des sciences t. 96 p. 441.1896 . 